\title{
Editorial
}

\section{New Advances in Distributed Control of Large-Scale Systems}

\author{
Dan Zhang, ${ }^{1}$ Wen-An Zhang, ${ }^{1}$ Zheng-Guang Wu, ${ }^{2}$ Kun Liu, ${ }^{3}$ \\ Hui Zhang, ${ }^{4}$ and Yun-Bo Zhao ${ }^{1}$ \\ ${ }^{1}$ Department of Automation, Zhejiang University of Technology, Hangzhou, China \\ ${ }^{2}$ The National Laboratory of Industrial Control Technology, Institute of Cyber Systems and Control, Zhejiang University, \\ Hangzhou, China \\ ${ }^{3}$ ACCESS Linnaeus Centre and School of Electrical Engineering, KTH Royal Institute of Technology, Stockholm, Sweden \\ ${ }^{4}$ The Merchant Marine College, Shanghai Maritime University, Shanghai, China
}

Correspondence should be addressed to Dan Zhang; danzhang@zjut.edu.cn

Received 30 August 2015; Accepted 31 August 2015

Copyright (C) 2015 Dan Zhang et al. This is an open access article distributed under the Creative Commons Attribution License, which permits unrestricted use, distribution, and reproduction in any medium, provided the original work is properly cited.

With the rapid development of the modern industry, the scale of process system of industry becomes more huge, the system functions are more complex, and the geographical distribution of system tends to be more distributed. Representative systems are chemical industrial network, robotic systems, smart grids, sensor networks, and so on [1-3]. The rapid development of industry system inevitably imposes much difficulty on the control system design. Traditional control system design theory cannot be applied to these large-scale systems. The distributed control system integrates control, computation, communication technology, and so forth and adapts to the developing trend of industrial control system. However, insertion of communication networks into the control systems comes at the price of nonideal signal transmission: the data sent through the networks experience time delay or suffer transmission data losses [4]. So far, research attention has been paid to this area; see [5-9] and the references therein.

In this issue, some research works on large-scale systems and the related topics are reported. We are proud to notice that these limited numbers of research works have shown a great variety and in a sense give a fairly complete picture of the state-of-the-art research on large-scale systems. In addition, a brief tutorial on large-scale systems from the Editorial Board is also included in this special issue.

(1) Theoretical studies on the large-scale multiagent systems are reported here. For example, J. Gao et al. discussed the consensus of multiagent systems, Y. Zhang et al. studied the two-dimension first-order multiagent systems, R. Wang et al. discussed the modeling and control of highly flexible solar-powered UAVs, and M. Huo et al. focus on the fault reconstruction approach for distributed coordinated spacecraft attitude control system.

(2) Theoretical analysis on the time-delay, packet dropouts in the large-scale networked systems are reported. For example, D.-M. Dai discussed the fault detection for networked systems based on the reduced-order filter, X. Lu et al. studied the fix-point smoothing for descriptor systems with multiplicative noise and single delayed observations, W. Yanfeng et al. considered the fault-tolerant control for networked control systems with limited information in case of actuator fault, S. Zhang et al. focus on the stabilization of networked distributed systems with partial and event-based couplings, and $\mathrm{Z}$. $\mathrm{Hu}$ and $\mathrm{Z}$. $\mathrm{Xu}$ give some solution on the distributed detection over a noisy multiaccess channel.

(3) Other results on the large-scale systems, such as microgrids, electric vehicle hydroturbine, and neural networks, are also discussed. Interested readers are referred to the detailed papers reported in this issue.

We hope this special issue will be a useful reference for people working on this area, and more fruitful results will be obtained in the time ahead based on the published works. 


\title{
Acknowledgment
}

We appreciate all the researchers for their valuable work on the large-scale systems.

\author{
Dan Zhang \\ Wen-An Zhang \\ Zheng-Guang Wu \\ Kun Liu \\ Hui Zhang \\ Yun-Bo Zhao
}

\section{References}

[1] J. B. Rawlings and B. T. Stewart, "Coordinating multiple optimization-based controllers: new opportunities and challenges," Journal of Process Control, vol. 18, no. 9, pp. 839-845, 2008.

[2] Y. Zheng, S. Li, and N. Li, "Distributed model predictive control over network information exchange for large-scale systems," Control Engineering Practice, vol. 19, no. 7, pp. 757-769, 2011.

[3] V. Sundarapandian, "Distributed control schemes for largescale interconnected discrete-time linear systems," Mathematical and Computer Modelling, vol. 41, no. 2-3, pp. 313-319, 2005.

[4] L. Zhang, H. Gao, and O. Kaynak, "Network-induced constraints in networked control systems-a survey," IEEE Transactions on Industrial Informatics, vol. 9, no. 1, pp. 403-416, 2013.

[5] S. C. Xu and J. Bao, "Distributed control of plant-wide chemical processes with uncertain time-delays," Chemical Engineering Science, vol. 84, pp. 512-532, 2012.

[6] F. Kazempour and J. Ghaisari, "Stability analysis of modelbased networked distributed control systems," Journal of Process Control, vol. 23, no. 3, pp. 444-452, 2013.

[7] M. Pajic, S. Sundaram, G. J. Pappas, and R. Mangharam, "The wireless control network: a new approach for control over networks," Institute of Electrical and Electronics Engineers. Transactions on Automatic Control, vol. 56, no. 10, pp. 23052318, 2011.

[8] P. Millán, L. Orihuela, C. Vivas, F. R. Rubio, D. V. Dimarogonas, and K. H. Johansson, "Sensor-network-based robust distributed control and estimation," Control Engineering Practice, vol. 21, no. 9, pp. 1238-1249, 2013.

[9] D. Zhang, R. Y. Ling, Q.-G. Wang, L. Yu, and Y. Feng, "Sensornetwork-based distributed stabilization of nonlinear large-scale systems with energy constraints and random sensor faults," Journal of the Franklin Institute, vol. 352, no. 8, pp. 3345-3365, 2015. 


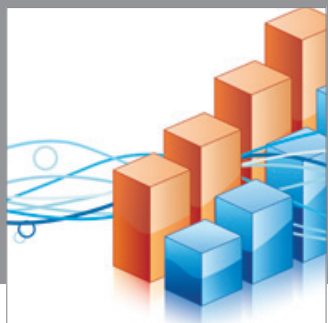

Advances in

Operations Research

mansans

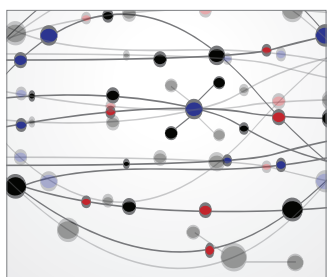

The Scientific World Journal
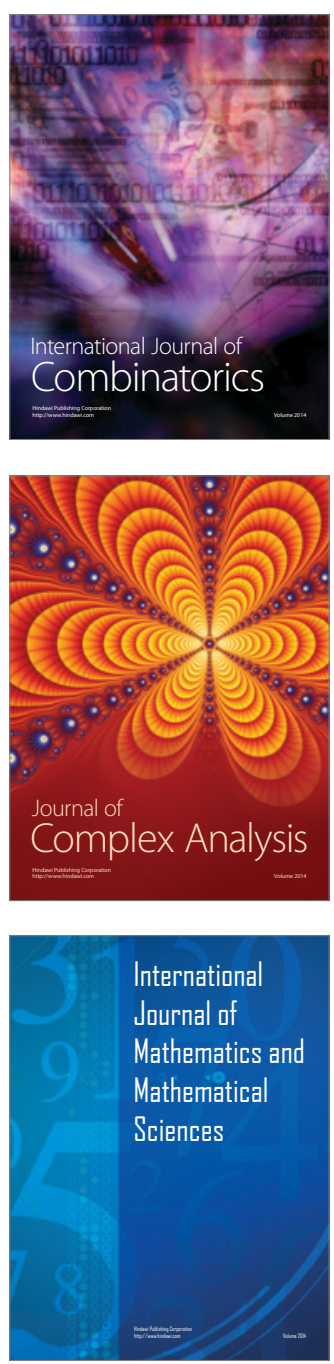
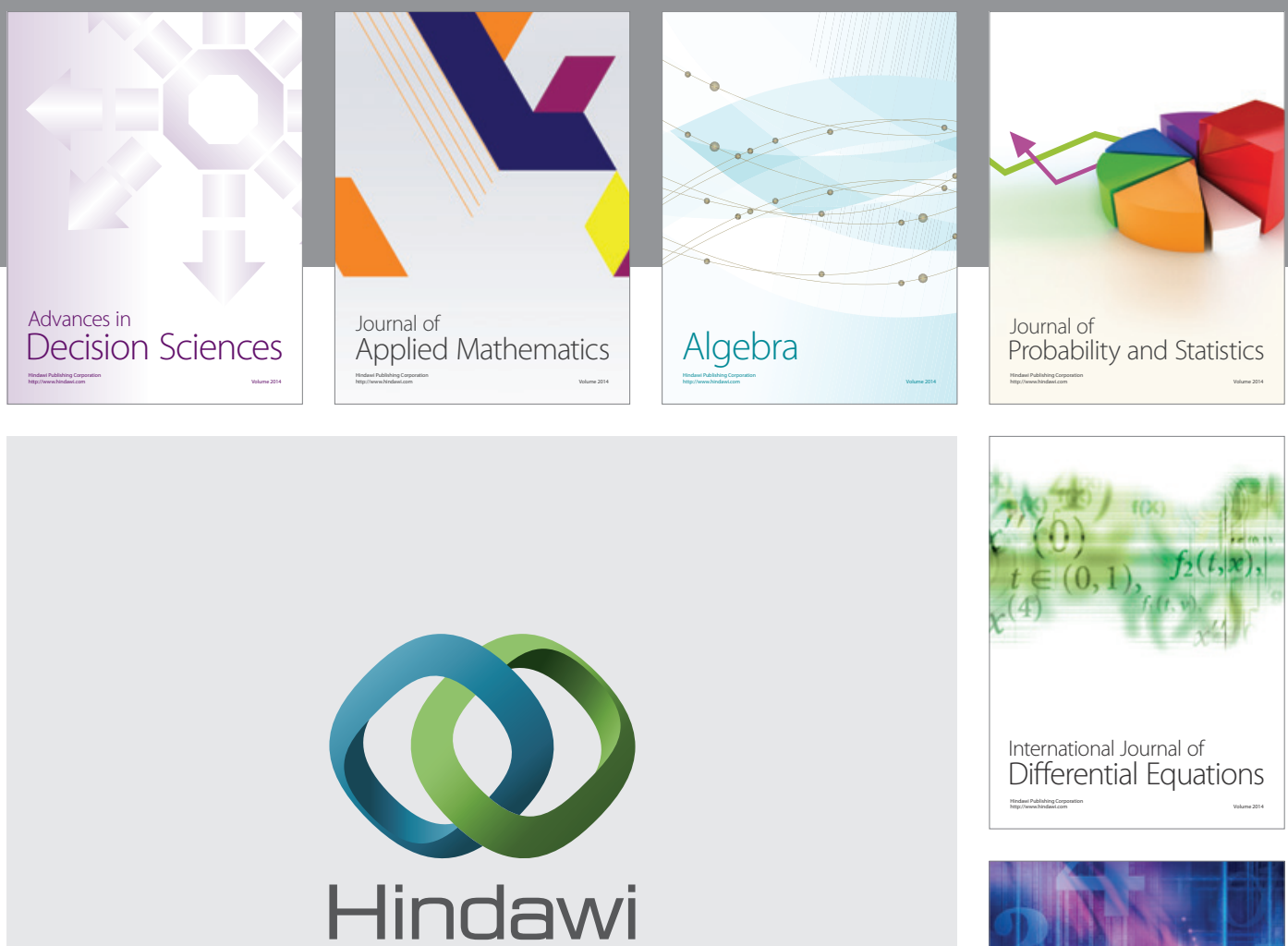

Submit your manuscripts at http://www.hindawi.com
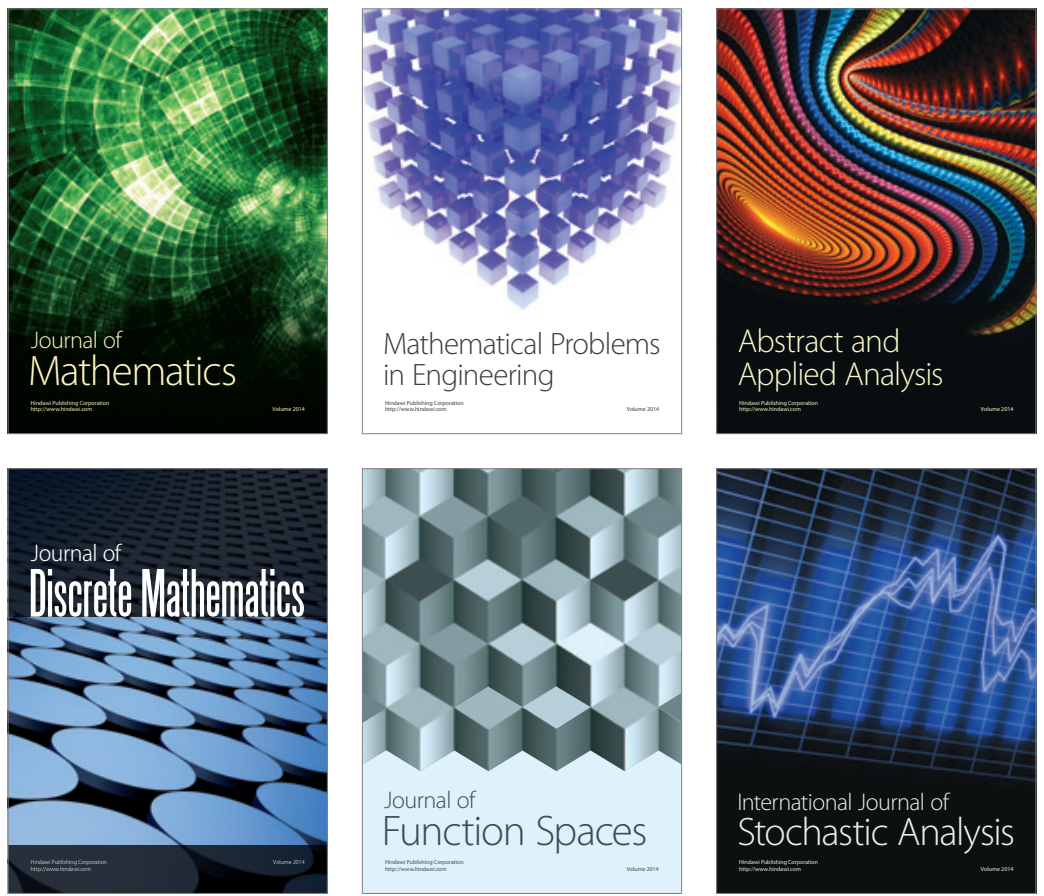

Journal of

Function Spaces

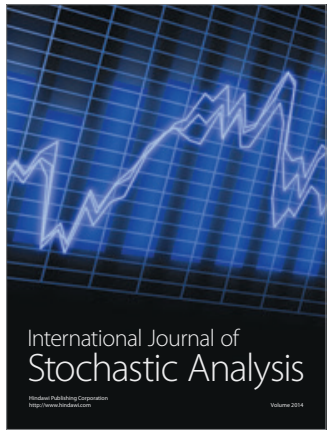

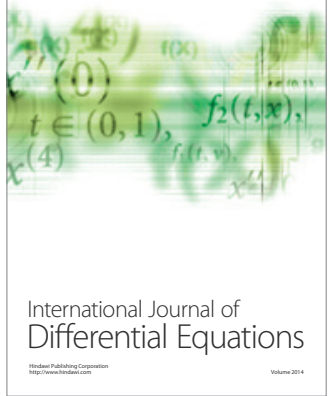
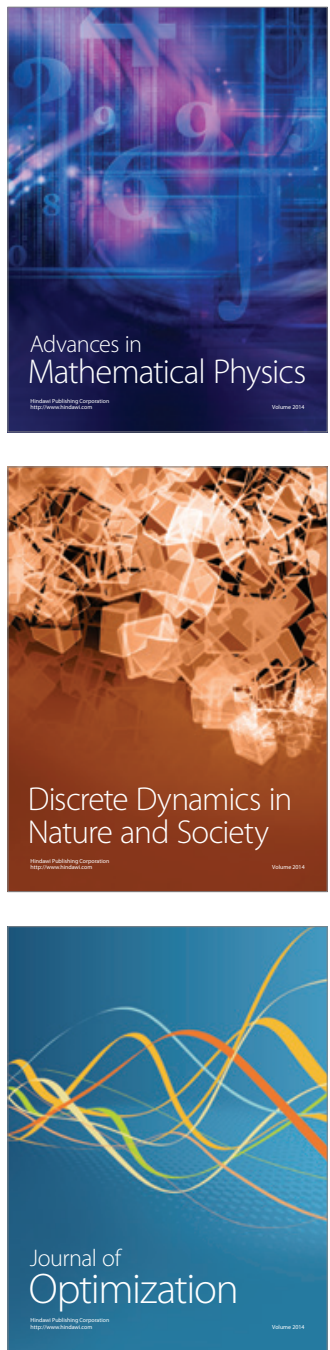